\title{
Plasma N-Terminal Pro-Brain Natriuretic Peptide Level was Lower in COPD Patients with Pulmonary Artery Hypertension
}

\author{
Zhao-Feng $\mathrm{Li}^{{ }^{*}}$, Qing-Lu Lin ${ }^{2}$, Qi-Feng Liu ${ }^{3}$, Yu-Zhen $\mathrm{Si}^{1}$, Bing-Guo Xue ${ }^{1}$, Yong-Qing wang $^{4}{ }^{*}$ \\ ${ }^{I}$ Department of Cardiology, No. 88 Hospital of PLA, Tai'an City, Shandong Province, 271000, China \\ ${ }^{2}$ Department of Anaesthesia, No. 88 Hospital of PLA, Tai'an City, Shandong Province, 271000, China \\ ${ }^{3}$ Department of Aeurosurgery, No. 88 Hospital of PLA, Tai'an City, Shandong Province, 271000, China \\ ${ }^{4}$ Department of Respiratory, No. 88 Hospital of PLA, Tai'an City, Shandong Province, 271000, China
}

*Corresponding Author: Zhao-Feng Li E-mail:lizhaofeng_2013@yahoo.com; Yong-Qing Wang Email: yongqingwang_1@163.com: The first three authours contribute equally to this work

\begin{abstract}
N-terminal pro-brain natriuretic peptide (NT-proBNP) is a pro-hormone, secreted by the myocardium in response to various stimuli, was found to be correlated with some hemodynamic parameters in pulmonary arterial hypertension (PAH). We investigated plasma NT-proBNP levels and the relationships between NT-proBNP and some hemodynamic parameters in chronic obstructive pulmonary disease (COPD) patients with or without PAH. We found that plasma NT-proBNP level was significantly lower in PAH group compared with the control group $(4982.5 \pm 22.5 \mathrm{pg} / \mathrm{ml}$ vs $5067.5 \pm 21.8 \mathrm{pg} / \mathrm{ml}, P<0.05)$. In the study population, only fasting insulin level was found to be positively correlated with fasting NT-proBNP level and only fasting insulin was an independent predictor of NT-proBNP (standardized coefficient $=0.708, P<0.001$ ) in a multiple regression model analysis. In the PAH group, only fasting insulin was found to be positively correlated with NT-proBNP, whereas other parameters were not found to be correlated with NT-proBNP. Our data suggests that the expression of NT-proBNP might be different in different kinds of pulmonary hypertension.
\end{abstract}

Keywords: NT-proBNP; COPD; pulmonary arterial hypertension

\section{INTRODUCTION}

Chronic obstructive pulmonary disease (COPD) is a very common disease. The global prevalence of COPD has been estimated to approximately $9-10 \%$ in populations aged 40 years or more [1]. Because of the development of severe hypoxemia, pulmonary arterial hypertension (PAH) develops late in the course of natural history of patients with COPD. The rate of PAH progression in COPD patients is slow (an increase of $<1 \mathrm{mmHg}$ per year) [2], however, the presence of PAH implies a poor prognosis in patients with COPD [3]. As it is known to us all, right heart catheterization (RHC) examination is the golden standard for the diagnosis of PAH, yet it is not practical to be used as a screening test. In the last few years, some researches have supplied with the fact that insulin, high density lipoprotein cholesterol, and C-reactive protein are all correlated with the severity of the patients with PAH $[4,5,6]$. In our previous study, we also found that circulating plasma ghrelin and obestatin levels were low in COPD patients with PAH [7].These circulating plasma markers could be used to value the severity of PAH and supply us with new pathways or therapeutic targets of PAH.

NT-proBNP is a pro-hormone, secreted by the myocardium in response to various stimuli, including mechanical stretch and hypoxia, along with some neurohormonal factors such as catecholamines and 
tumor necrosis factors. In 2008, Allanore and his colleagues first explored the plasma level of NTproBNP in scleroderma patients with PAH and supplied with the fact that NT-proBNP could be a biomarker to value the severity of PAH associated with scleroderma [8]. FIJALKOWSKA et al also found that circulating plasma NT-proBNP levels were correlated with echocardiographic variables and hemodynamic parameters in a heterogeneous cohort of pulmonary hypertension patients [9]. In 2012, Charlotte U. Andersen found that there was a significant relationship between higher values of NTproBNP and the presence of PAH in patients with interstitial lung disease [10]. The above results suggested that NT-proBNP might play a role in the regulation of pathophysiological process of PAH. The aim of this study was to investigate the plasma NT-proBNP levels and their relationships between them and some hemodynamic parameters in COPD patients with or without PAH.

\section{METHODS}

\subsection{Subjects}

Thirty-nine patients, ranging in age from 55 to 84 years old (mean age: $69.6 \pm 1.6$ years ), were recruited from those who were diagnosed COPD between October 2013 and February 2014. All patients had no evidences of liver or kidney diseases based on the laboratory examinations. These COPD patients were taken to the ultrasound department for echocardiography according to the guideline of ESC [11]. The estimation of systolic pulmonary arterial pressure (PASP) is based on the peak velocity of the jet of tricuspid regurgitation [11]. Based on the results of echocardiography, the patients were divided into two groups: twenty-two patients were enrolled in the PAH group (PASP $>50 \mathrm{mmHg}$ ), the rest seventeen patients were enrolled in the control group (PASP $<36 \mathrm{mmHg}$ ). Written informed consents were obtained from all subjects before participation.

\subsection{Anthropometry}

Height and weight were measured according to the standard techniques in each subject [12]. Body mass index (BMI) was calculated.

\subsection{Echocardiography}

All the patients were examined at rest in the supine position or left lateral decubitus position. A complete M-mode and 2D echocardiography study was performed by a senior sonographer using a standardized protocol on the same machine (Vivid 7, Vingmed-General Electric, Horten, Norway) according to the American Society of Echocardiography guidelines [13]. The left ventricular contractility (fractional shortening, FS and ejection fraction, EF) was measured by M-mode echocardiography performed from the parasternal long-axis view.

\subsection{Blood Samples}

Blood samples were taken from the femoral vein after an overnight fast. The blood was immediately transferred into a chilled glass tube containing EDTA-2Na $(1 \mathrm{mg} / \mathrm{ml})$ and aprotinin (Phoenix Pharmaceuticals, Belmont, CA; 100ul containing 0.6 trypsin inhibitor units per milliliter of blood), then immediately centrifuged at $1600 \times \mathrm{g}$ for 15 minutes at $4^{\circ} \mathrm{C}$. Plasma samples were frozen and stored at $-80^{\circ} \mathrm{C}$ until assayed. The plasma glucose levels were measured by an automated glucose oxidase method (Automatic Analyzer 7600-020; Hitachi, Tokyo, Japan).

\subsection{Hormone Assay}

Plasma NT-proBNP and insulin levels were measured by enzyme linked immunosorbent assay (ELISA) method according to the manufacturer's instructions. The reagent kits were manufactured by R\& D Systems (Minneapolis, USA). Insulin resistance was calculated by the homeostasis model of assessment for insulin resistance (HOMA-IR) approach, calculated as fasting insulin (microunits per mil-liter) $\times$ fasting blood glucose (millimoles per liter)/22.5 [14] 


\subsection{Statistical Analysis}

Data are expressed as the mean \pm SE. Comparisons between the parameters of the PAH group and the control group were performed with unpaired Student's $t$ test. The relationships between NTproBNP, insulin, and hemodynamic parameters were examined by bivariate correlations (Pearson's correlation coefficient). Multiple regression analysis was further used to assess the relationships between NT-proBNP, insulin and hemodynamic variables. A value of $\mathrm{P}<0.05$ was considered significant. All of the analyses were performed using SPSS for windows (version 10.0; SPSS Inc., Chicago, IL).

\section{ReSUlts}

\subsection{Subjects (Table 1)}

Table 1 depicts characteristics of the study population. There were no significant differences in BMI, age, fasting glucose and insulin levels between the PAH group and the control group. Compared with the control group, the PAH group had lower EF and fasting insulin level. However, the insulin resistance as calculated by the HOMA-IR approach was not found to show significant difference between the PAH group and the control group.

Table1. Characterization of the subjects included in the study

\begin{tabular}{|l|l|l|l|}
\hline Parameters & PAH group & Control group & P value \\
\hline Number & 22 & 17 & \\
\hline Age $(\mathrm{y})$ & $69.1 \pm 2.2$ & $70.3 \pm 2.4$ & 0.72 \\
\hline BMI $\left(\mathrm{Kg} / \mathrm{m}^{2}\right)$ & $20.1 \pm 0.75$ & $20.4 \pm 1.2$ & 0.80 \\
\hline EF & $0.60 \pm 0.01$ & $0.65 \pm 0.01$ & 0.03 \\
\hline Fasting glucose $(\mathrm{mmol} / \mathrm{liter})$ & $5.5 \pm 0.23$ & $5.1 \pm 0.18$ & 0.19 \\
\hline Fasting insulin $(\mu \mathrm{Iu} / \mathrm{ml})$ & $10.3 \pm 0.14$ & $11.0 \pm 0.14$ & 0.002 \\
\hline HOMA-IR & $2.49 \pm 0.11$ & $2.47 \pm 0.10$ & 0.88 \\
\hline
\end{tabular}

Data are means \pm SE and $P<0.05$ was regarded as statistically significant.

\subsection{Differences in NT-proBNP level between the PAH group and the control group}

The fasting plasma concentration of NT-proBNP was $4982.5 \pm 22.5 \mathrm{pg} / \mathrm{ml}$ and $5067.5 \pm 21.8 \mathrm{pg} / \mathrm{ml}$ in PAH group and control group, respectively, and there was a significant difference between them $(\mathrm{P}<0.05)$ (Fig. 1).

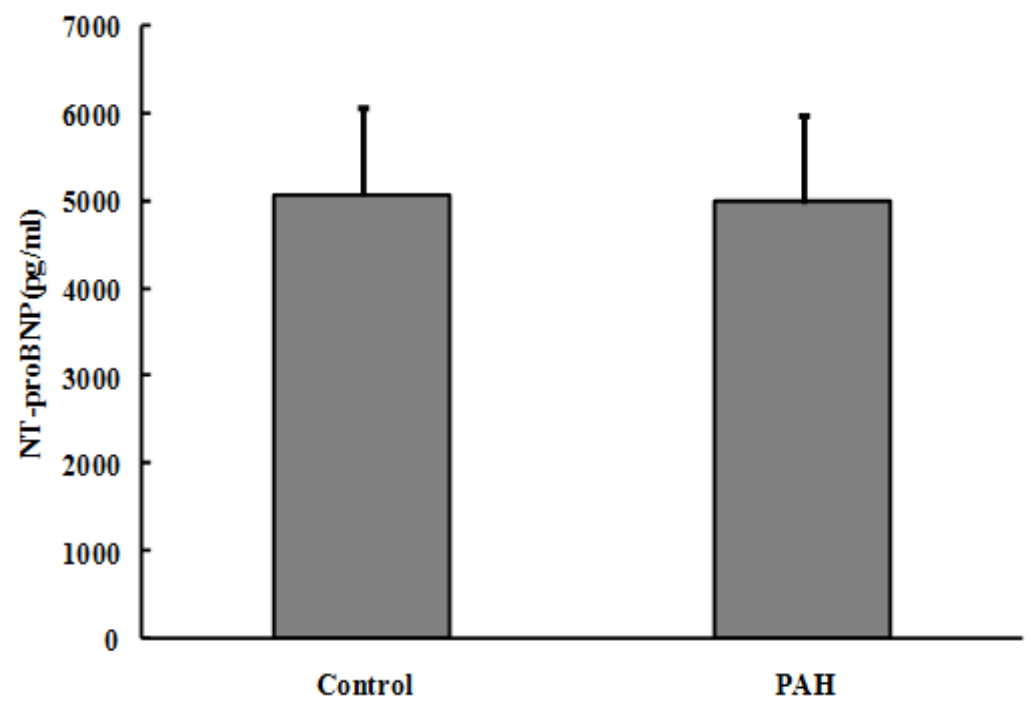

Fig1. Plasma NT-proBNP levels in PAH and control group. $* P<0.05$ compared with control group. 


\subsection{Correlations between NT-proBNP and Other Parameters of the Baseline in the Study Population}

In the study population, only fasting insulin level was found to be positively correlated with fasting NT-proBNP level. Age, BMI, EF, fasting glucose, and HOMA-IR were not found to be correlated with NT-proBNP. In a multiple regression model including age, BMI, fasting glucose, fasting insulin, HOMA-IR, EF, only fasting insulin was an independent predictor of NT-proBNP (standardized coefficient $=0.708, \mathrm{P}<0.001$ ), whereas other parameters did not show any significant correlations with NT-proBNP. In the PAH group, only fasting insulin was found to be positively correlated with NT-proBNP, whereas other parameters were not found to be correlated with NT-proBNP. In the control group, NT-proBNP was not found to be significant correlated with any parameters and no parameter was found to be an independent predictor of NT-proBNP in a multiple regression model analysis.

Table2. Correlations between NT-proBNP and hemodynamic parameters in the study population

\begin{tabular}{|l|l|l|l|l|l|l|}
\hline \multicolumn{1}{|c|}{ Parameters } & \multicolumn{2}{|l|}{ All the patients $(\mathrm{n}=39)$ in } & \multicolumn{2}{l|}{ PAH group $(\mathrm{n}=22)$} & \multicolumn{2}{l|}{ Control group $(\mathrm{n}=17)$} \\
\hline & $\mathrm{r}$ & $\mathrm{p}$ & $\mathrm{r}$ & $\mathrm{p}$ & $\mathrm{r}$ & $\mathrm{P}$ \\
\hline Age & 0.106 & 0.261 & 0.373 & 0.087 & -0.354 & 0.163 \\
\hline BMI & 0.004 & 0.491 & -0.153 & 0.497 & 0.138 & 0.598 \\
\hline Fasting glucose & -0.076 & 0.323 & -0.085 & 0.708 & 0.226 & 0.383 \\
\hline Fasting insulin & 0.708 & $<0.001$ & 0.565 & 0.006 & 0.776 & 0.0003 \\
\hline HOMA-IR & 0.176 & 0.142 & 0.077 & 0.733 & 0.433 & 0.083 \\
\hline EF & 0.156 & 0.171 & -0.152 & 0.498 & 0.314 & 0.220 \\
\hline
\end{tabular}

\section{DISCUSSION}

The aim of the present study was to investigate the plasma NT-proBNP level and clinical hemodynamic parameters and their relationships in COPD patients with and without PAH. In this study, we found that fasting plasma NT-proBNP level was significantly lower in COPD patients with PAH compared with that of the control group.

In a previous study, plasma BNP level was found to be elevated in patients with PAH and chronic lung disease with right ventricular overload [15]. In a study enrolled in 44 patients with pulmonary hypertension Nagaya et al. showed a significant difference between those with pulmonary hypertension compared to those without regarding their BNP levels [16]. In 2006, Williams MH et al found that plasma NT-proBNP level was significantly higher in PAH patients associated with systemic sclerosis (PAH-SSc) compared with the SSc patients without PAH [17]. Recently, Mathai SC et al. also found that plasma NT-proBNP level was significantly higher in PAH-SSc compared with idiopathic PAH (IPAH) patients and was a stronger predictor of survival in PAH-SSc than IPAH [18]. The results of Mathai's group suggested that the expression of NT-proBNP might be different in the different kinds of PAH. Therefore, this is the aim to explore the expression of NT-proBNP and the relationships between it and some hemodynamic parameters in the COPD patients with and without PAH.

In this study, we found that $\mathrm{EF}$ was lower in the PAH group compared with that of the control group and the difference between them was significant. Recently, EF was also found to be significant lower in stable COPD patients compared with that of the control [19]. Based on the above results, we might speculate that left ventricular systolic dysfunction was more serious in COPD patients with PAH compared with that of the control group.

In this study, we also explored the relationships between NT-proBNP and some hemodynamic parameters in COPD patients. We found that only fasting insulin level was positively correlated with NT-proBNP in the study population and only insulin was an independent predictor of NT-proBNP in a multiple regression model analysis. In the PAH group, we also found fasting insulin level was not 
only positively correlated with NT-proBNP but also an independent predictor of NT-proBNP. However, the same correlations between NT-proBNP and insulin were not found in the control group.

In this study, we also found that fasting insulin level was lower in COPD patients with PAH compared with that of control group, and the difference between them was significant. However, the insulin resistance calculated by the HOMA-IR approach of the PAH group was higher compared with that of the control group and the difference between them was not significant. This was not in accordance with the previous study of Zamanian RT's group in 2008 which revealed that insulin resistance was more prevalent in patients with PAH [6].

There were several limitations in this study. First, the results of this study should be tested in a larger sample of patients where subgroup comparisons could be allowed (i.e. female vs male, different levels of PAP). Second, a study with a longer follow-up period would supply us with more information between NT-proBNP and clinical hemodynamic parameters and determine whether NT-proBNP could be used as a biomarker of severity of PAP in the COPD patients with PAH. Finally, we did not supply more evidences to explain the differences between the previous studies and our study.

\section{CONCLUSION}

In conclusion, the aim of this study was to explore the expression of NT-proBNP and the relationships between it and some hemodynamic parameters in COPD patients with or without PAH. The results of this study suggested that the expression of NT-proBNP might be different in different kinds of pulmonary hypertension. The difference of the results between the previous and our study might be due to the different enrolled patients in different studies. Future studies to confirm or refute our initial results are eagerly anticipated.

\section{REFERENCES}

[1] Halbert RJ, Natoli JL, Gano A, Badamgarav E, Buist AS, et al. Global burden of COPD: systematic review and meta-analysis. Eur Respir J 2006; 28:523-532.

[2] Weitzenblum E, Sautegeau A, Ehrhart M, Mammosser M, Hirth C, et al. Long-term course of pulmonary arterial pressure in chronic obstructive pulmonary disease. Am Rev Respir Dis 1984; 130:993-998.

[3] Weitzenblum E, Hirth C, Ducolone A, Mirhom R, Rasaholinjanahary J, et al. Prognostic value of pulmonary artery pressure in chronic obstructive pulmonary disease, Thorax 1981;36: 752-758.

[4] Heresi GA, Aytekin M, Newman J, DiDonato J, Dweik RA. Plasma levels of high-density lipoprotein cholesterol and outcomes in pulmonary arterial hypertension. Am J Respir Crit Care Med 2010; 182: 661668.

[5] Quarck R, Nawrot T, Meyns B, Delcroix M. C-reactive protein: a new predictor of adverse outcome in pulmonary arterial hypertension. J Am Coll Cardiol 2009; 53: 1211-1218.

[6] Zamanian RT, Hansmann G, Snook S, Lilienfeld D, Rappaport KM, et al. Insulin resistance in pulmonary arterial hypertension. Eur Respir J 2009; 33:318-324.

[7] Li ZF, Li YP, Chen JS, Xue BG, Yu-Zhen Si, et al. Circulating plasma ghrelin and obestatin levels are low in chronic obstructive pulmonary disease patients with pulmonary artery hypertension. International Journal of Research Studies in Biosciences 2015; 3:67-72.

[8] Allanore Y, Borderie D, Avouac J, Zerkak D, Meune C, et al. High N-terminal pro-brain natriuretic peptide levels and low diffusing capacity for carbon monoxide as independent predictors of the occurrence of precapillary pulmonary arterial hypertension in patients with systemic sclerosis. Arthritis Rheum 2008; 58: 284-291.

[9] Fijalkowska A, Kurzyna M, Torbicki A, Szewczyk G, Florczyk M, et al. Serum N-terminal brain natriuretic peptide as a prognostic parameter in patients with pulmonary hypertension. Chest 2006; 129: $1313-1321$. 
[10] Charlotte U. Andersen, Søren Mellemkjær, Jens Erik Nielsen-Kudsk, Elisabeth Bendstrup, et al. Diagnostic and prognostic role of biomarkers for pulmonary hypertension in interstitial lung disease. Respiratory Medicine 2012; 106: 1749e-1755e.

[11] Grünig E, Barner A, Bell M, Claussen M, Dandel M, et al. Non-invasive diagnosis of pulmonary hypertension: ESC/ERS Guidelines with Updated Commentary of the Cologne Consensus Conference. Int J Cardiol 2011; 154:S3-S12.

[12] Chen C, Lu FC. The guidelines for prevention and control of overweight and obesity in Chinese adults. Biomed Environ Sci 2004; 17:1-36.

[13] Cheitlin MD, Armstrong WF, Aurigemma GP, Beller GA, Bierman FZ, et al. ACC/AHA/ ASE 2003 guideline update for the clinical application of echocardiography-summary article: a report of the American College of Cardiology/American Heart Association Task Force on Practice Guidelines (ACC/AHA/ASE Committee to Update the 1997 Guidelines for the Clinical Application of Echocardiography). J Am Coll Cardiol 2003; 42:954 -970.

[14] Matthews DR, Hosker JP, Rudenski AS, Naylor BA, Treacher DF, et al. Homeostasis model assessment: insulin resistance and beta-cell function from fasting plasma glucose and insulin concentrations in man. Diabetologia 1985; 28:412-419.

[15] Bando M, Ishii Y, Sugiyama Y, Kitamura S. (1999) Elevated plasma brain natriuretic peptide levels in chronic respiratory failure with cor pulmonale. Respir Med. 93:507-514.

[16] Nagaya N, Nishikimi T, Uematsu M, Satoh T, Kyotani S, et al. (2000) Plasma brain natriuretic peptide as a prognostic indicator in patients with primary pulmonary hypertension. Circulation 102:865-870.

[17] Williams MH, Handler CE, Akram R, Smith CJ, Das C, et al. (2006) Role of N-terminal brain natriuretic peptide (N-TproBNP) in scleroderma-associated pulmonary arterial hypertension. Eur Heart J 27:14851494.

[18] Mathai SC, Bueso M, Hummers LK, Boyce D, Lechtzin N, et al. (2010) Disproportionate elevation of Nterminal pro-brain natriuretic peptide in scleroderma-related pulmonary hypertension. Eur Respir J 35:95104.

[19] Gehan H. AboEl-Magd, Timoor Hassan, Mohamed H. Aly, Maaly M. Mabrouk. (2017) Echocardiography and N-terminal-pro-brain natriuretic peptide in assessment of left ventricular diastolic dysfunction in stable COPD in relation to disease severity. Egyptian Journal of Chest Diseases and Tuberculosis 66:75-80.

Citation: Z. Feng Li, "Plasma N-Terminal Pro-Brain Natriuretic Peptide Level was Lower in COPD Patients with Pulmonary Artery Hypertension", International Journal of Research Studies in Biosciences (IJRSB), vol. 5, no. 9, pp. 16-21, 2017. http://dx.doi.org/10.20431/2349-0365.0509004

Copyright: (C) 2017 Authors. This is an open-access article distributed under the terms of the Creative Commons Attribution License, which permits unrestricted use, distribution, and reproduction in any medium, provided the original author and source are credited. 